\title{
Magnetic field transport from AGN cores to jets, lobes, and the IGM
}

\author{
Philipp P. Kronberg ${ }^{1} \dagger$ \\ Department of Physics, University of Toronto, Toronto, ON M5S 1A7, Canada \\ email: kronberg@physics.utoronto.ca
}

\begin{abstract}
I describe various stages of energy flow along an extragalactic jet, which subsequently evolves into an extended lobe which is visible in radio and X-rays. The sizes of the lobes vary from kpc scales to several megaparsec, so that the largest lobes are clearly injecting back hole energy into the IGM on scales comparable with a galaxy-galaxy separation. This is sometimes loosely referred to as Black hole-IGM "feedback". My talk begins with a well-formed jet, and avoids the complex and unclarified physics at less than a few Schwarzschild radii that cause the initial launching the jet.

This presentation focuses on recent thinking and supercomputer simulations that appear to clarify the fundamental nature of these remarkable jets and lobes. The energy transport process appears to be electrodynamic, rather than particle beam-driven. A new observational verification of a $10^{18}$ Ampère current in an actual jet is concordant with the predictions and simulations of poynting flux-dominated electromagnetic jets. In this model the current is tightly related to the $\mathrm{BH}$ mass and angular energy.

The magneto-plasma properties of the lobes must obviously match to the jets which feed them. The "energy sink" phase is when BH energy is ultimately deposited on supra-galactic scales. The process from the BH to the lobe production happens with remarkable efficiency. The presence or absence of a galaxy cluster environment creates laboratory conditions that help to calibrate the energy flow paths, and the magnetic rigidity of these jet-lobe systems.

I conclude by describing recent, sensitive radio observations on supra-cluster scales that test for final magnetic energy deposition - the "sink" phase - into the intergalactic medium.
\end{abstract}

Keywords. Galaxies: jets - galaxies: active - galaxies: magnetic fields - intergalactic medium

\section{The nearby, black hole-driven jet in M87}

Our ability to diagnose extragalactic radio jets is currently limited by instrumental sensitivity, and especially by limited resolution transverse to the jet. Currently, jets can be probed on scales of a few parsecs with very long baseline interferometry (VLBI), and on scales above $\sim 100$ parsecs with connected interferometers such as the NRAO VLA. The best $\mathrm{cm} \lambda$ VLBI images have been made at $\sim 10^{-3}$ arcsec resolution. The VLA's resolution is typically $\sim 0.1$ arcsec or larger. Figure 1 shows state-of-the-art images of the M87 jet in each resolution range.

At only $15 \mathrm{Mpc}$ distance, M87 is the nearest "laboratory" jet, emanating from a nuclear $10^{9} \mathrm{M}_{\odot}$ black hole. By common intergalactic standards M87, despite its high BH mass, is a relatively small and low luminosity radio galaxy.

It is evident that in both régimes of angular resolution in Figure 1, the best available resolution at $\mathrm{cm}$ wavelengths fails to show the brightest jet features with high resolution across the jet. To study the jet in the desired detail, the transverse resolution should ideally be $\sim 10$ times higher, and at several wavelengths so that the Faraday rotation

$\dagger$ Present address: Los Alamos National Laboratory P.O Box 1663, MS T006, Los Alamos NM 87545, USA 

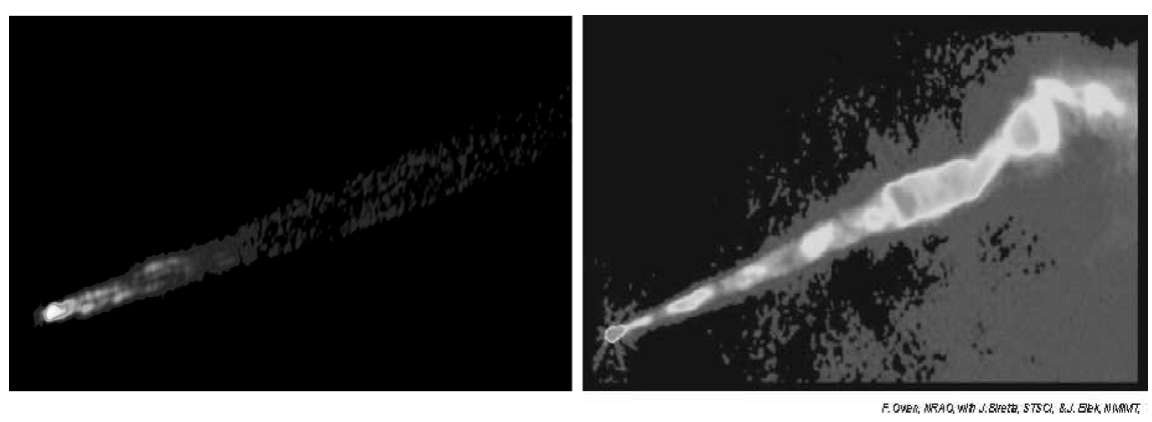

Figure 1. Left: VLBI image of the inner 0.01 arcsec of the M 87 jet at a resolution of $0.8 \times 1.3$ milliarcsec (Kovalev et al., 2007). Right: A VLA $15 \mathrm{GHz}$ image of a 15 arcsec jet segment at a resolution of 0.1 arcsec Owen et al. 1989).

and polarization structure can be well studied. Yet M87 is one of the most easily "accessible" jets because of its unique proximity. This illustrates why extragalactic jets have not yielded easy answers about their detailed physics over the past four decades. Unfortunately there is little immediate prospect of exceeding the VLA's resolution at the required $\mathrm{cm}-\lambda$ wavelength range. However increasing interferometer capability down to $\lambda \sim 3 \mathrm{~mm}(\sim 90 \mathrm{GHz})$ will partially improve this resolution deficiency. Partially, because higher resolution is also required at the longer $\mathrm{cm}$ wavelengths where Faraday rotation is needed to probe the plasma parameters. For kpc scale jets, the plasma parameters are such that sufficient Faraday depths occur mainly at the longer wavelengths, as will be illustrated in the discussion of 3C303 below.

\section{3C303 a kpc-scale jet at $z=0.141$}

The moderately distant 3C303 offers is a more typical example of a radio galaxy in terms of its radio luminosity and dimensions. Figure 2 shows the the central $\sim 1 / 3$ of the radio image, where we see a repeating "knot" structure similar to M87, and seen in several other kpc-scale jets. However the knot lengths are about 20 times those of M87's knots, and their radiating volumes are $\sim 10^{4}$ times those in M87. This illustrates, as does Figure 1, that the magneto-plasma structures in these jets are a highly scalable phenomenon; The linear extents of the largest, "giant" radio galaxies (GRG) are a further factor of $\sim 20$ bigger than 3C303.

3C303 originally attracted interest because of its somewhat unusual morphology (Kronberg 1976a,b, 1986, Leahy \& Perley 1991), and its unusual optical field (Kronberg et al. 1977). It had been imaged in I, Q, and U in 2 bands with the NRAO Green Bank Interferometer in 1970/71 (Kronberg, 1976b) and in 3 bands (1.4, 6 and $15 \mathrm{GHz}$, each in Stokes I, Q, and U) in 1981 with the newly completed VLA (Kronberg, 1986; see also Leahy \& Perley, 1991). In an X-ray study of this system, Kataoka et al. (2003) found that the Chandra X-ray image of 3C303 has remarkable morphological similarities to the VLA $1.4 \mathrm{GHz}$ image, including the jet "knots", and at the similar resolution of $\sim 2$ ".

It has become apparent meanwhile that 3C303 shares common properties with many AGN-jet radio galaxies and quasars (see Bridle et al., 1994). Its magnetic and plasma properties discussed below should be regarded as probably typifying a broad class of extragalactic jet systems.

\subsection{Jet plasma parameters}

The synchrotron luminosity of 3C303's jet, its associated equipartition field strength, the overall source luminosity, the regular magnetic field structure in the knots, lack of 


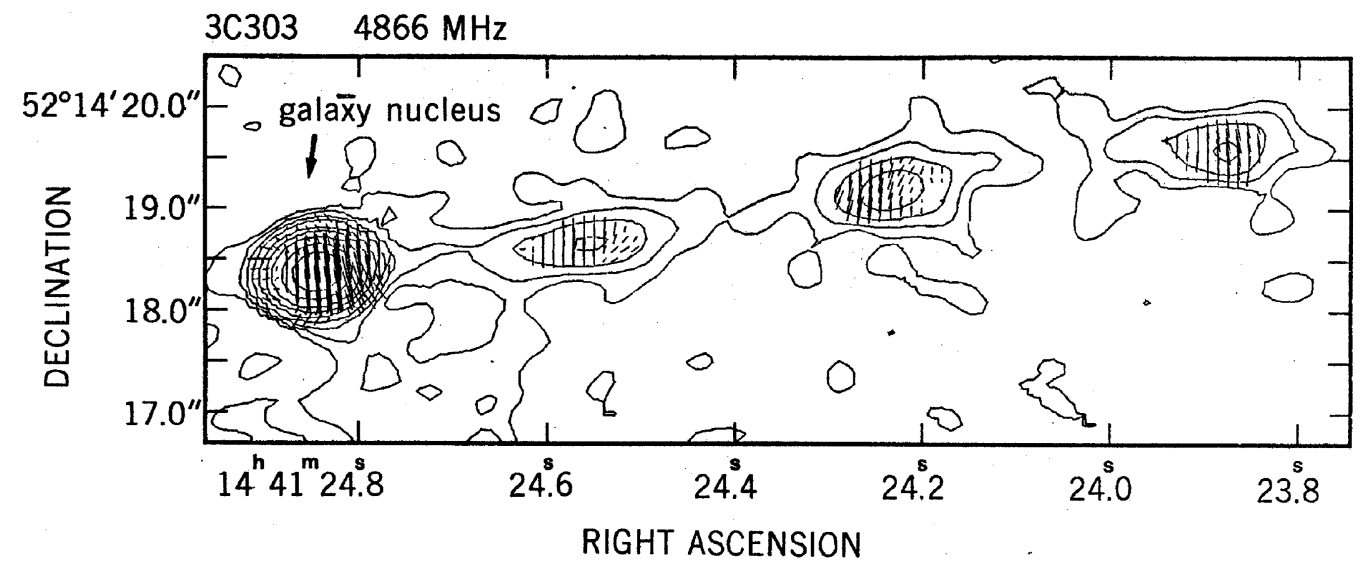

Figure 2. A VLA image of 3C303 at $\lambda 6 \mathrm{~cm}$ in Stokes' parameters I, Q, and U. degree of polarization in the knots is high, of order $20 \%$, and the polarization structure tends to repeat from knot to knot (Lapenta \& Kronberg, 2005).

depolarization in the knots, and low depolarization between $\lambda \lambda 6$ and $20 \mathrm{~cm}$ in the knots, are informative for constructing and testing physical models of the jet.

Details of the analysis methods for 3C303 are given in Lapenta and Kronberg (2005), and are summarized in the following results: The total source luminosity from $\sim 100$ $\mathrm{MHz}$ to $\sim 10 \mathrm{keV}$ is $\sim 1.7 \times 10^{42} \mathrm{erg} \mathrm{s}^{-1}$. The equipartition-assumed jet magnetic field strength at $\simeq 400 \mathrm{pc}$ from the jet axis is $|\mathrm{B}| \sim 3 \mathrm{mG}$. This, combined with the Faraday rotation and depolarization limits gives $n_{t h} \leqslant 1.4 \times 10^{-5} \mathrm{~cm}^{-3}$ within the knot zones around the jet. The combination of $|\mathrm{B}|$ and $n_{t h}$ gives an approximate lower limit to the Alfvén speed within the knot volumes, which is a significant fraction of $c$.

$$
V_{A}^{k n o t} \propto \frac{B^{k n o t}}{\sqrt{n_{t h}}} \approx c
$$

This means that the plasma $\beta=n_{t h} k \mathrm{~T}_{8} /\left(\mathrm{B}^{2} / 8 \pi\right)$ is very low $\left(\leqslant 10^{-5}\right), \mathrm{T}_{8}$ being the plasma temperature in units of $10^{8} \mathrm{~K}$. The correspondingly low $n_{t h}$ also suggests that the 1- $5 \mathrm{keV}$ X-ray emission from the knots cannot be thermal bremsstrahlung, nor IC emission because of the $\mathrm{mG}$ level magnetic field, and must, solely on these arguments, be $\mathrm{X}$-ray synchrotron radiation. The highly polarized radio synchrotron radiation indicates a well-organized magnetic field. It is consistent with, thought does not prove, a low pitch angle helical structure about the jet axis.

\subsection{First assumption-free measurement of an extragalactic jet current}

A particular magnitude and direction of a Faraday rotation measure gradient can, in the right circumstances, indicate an electric current. The resolved knot "C", third from the AGN in Figure 2 shows a RM gradient $(\nabla \mathrm{RM})$, a $2-\mathrm{D}$ vector that is exactly perpendicular to the jet axis. This would correspond to a net current, within a particular radius from the jet axis, if, and only if, $\mathrm{RM}=0$ exactly on the jet axis. To test this requires an absolute calibration of RM in the vicinity of 3C303's jet. This has recently been possible with a new large independent sample of extragalactic radio source RM's close to the $(l, b)$ location of $3 \mathrm{C} 303$ where, in fact, the RM sky is relatively "quiet". Indeed RM $\approx$ 0 within measurement errors after subtracting the smoothed RM of nearby background 

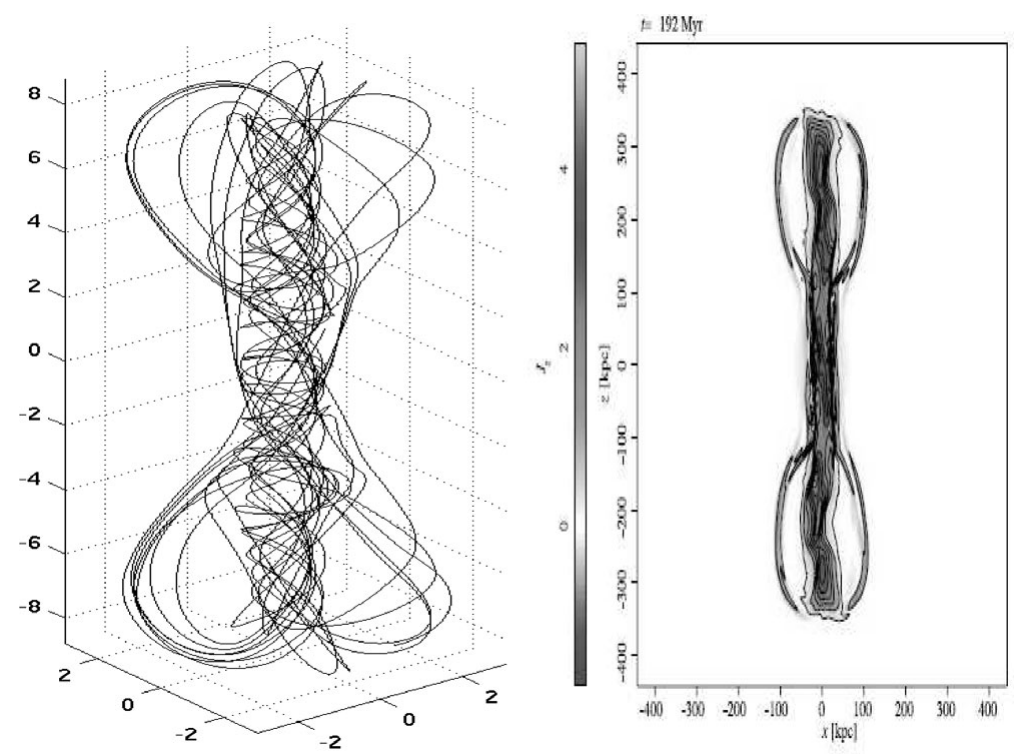

Figure 3. Left: The helical structure of a simulated "magnetic tower" poynting flux jet, after Li et al. (2006). Right: Simulated current structure of a combined magnetic tower poynting flux jet + lobe system (Nakamura et al., 2008).

sources. Adding this information to the above indicates that the 3C303 jet has an average current within $\simeq 400 \mathrm{pc}$ of its axis, which is $7 \times 10^{17}$ ampères, and directed away from the central BH (AGN) (Ji et al., 2008). It is the largest electrical current ever measured. This result is of particular interest in the context of poynting flux energy flow models for extragalactic jets, as we shall discuss in the next section.

\section{Poynting flux jets}

The idea that the jet power from a rotating black hole is delivered not via a particle beam, but electromagnetically, was proposed by Lovelace (1976), and developed in several succeeding papers: Blandford \& Znajek (1977), Benford (1978), Lesch, Appl \& Camenzind (1989) and others.

The extraction of this energy from the central BH involves complex magneto-plasma processes, and possibly the coupling into the $\mathrm{BH}$ rotational energy at the ergosphere in a Kerr metric-distorted space-time. Partly due to lack of access to observation, the physics of the jet launching at this level has not yet been completely clarified. However the already-formed jet better lends itself to modeling, analysis and observation as illustrated above. A considerable literature exists on jets and their confinement, and in this limited space I concentrate solely on the class of electromagnetic, poynting flux jets. Why? because impressive recent progress has been made in understanding the relevant magnetoplasma processes, and in their simulation. Figure 3 shows a supercomputer simulation by Li et al. (2006) of an electromagnetically dominated jet and of its current structure (Nakamura et al. 2008).

In the electromagnetic model the jet current is coupled to the BH. It is integral to both the stability, and instabilities in the jet and lobe. The jet and lobe are part of the same magneto-plasma system. The magnitude of $\sim 10^{18}$ ampères for the electric current associated with a $10^{8} \mathrm{M}_{\odot} \mathrm{BH}$, was originally predicted by Lovelace (1976). It is integral 
to the simulations of Li et al. (2006) in Figure 3, and now tentatively confirmed in an actual measurement in (by Kronberg in Ji et al., 2008) as discussed above.

Another success of this class of model is that the current-driven instabilities are more able than hydrodynamic, e.g. Kelvin-Helmholz, instabilities to recover at locations of instability and still retain the energy in the jet-lobe "system", rather than to scatter and dissipate the power. This robustness to complete disruption appears both observationally and theoretically confirmed in the multi-bubble (lobe) system in the Hydra A cluster(Wise et al., 2007, Diehl et al., 2008). Here the jet energy flow appears to be "pinched" at the "end" of one lobe, only to re-appear and energize a successive coherent lobe! Such behaviour appears only to be possible if the jet and lobe are magnetically dominated, and maintained by a (current driven) reverse field pinch process (e.g. Benford \& Protheroe 2008).

The electromagnetic jet is likely to push shocked ambient ahead of it, causing shockinduced particle acceleration and a visible outer lobe beyond the magnetic structure in Fig 3a. This outer shock structure is broadly similar to what is predicted in particle beam/hydrodynamic models, and this has tended to mask the observational evidence for poynting flux dominated power flow. The jet's visibility in synchrotron radiation is expected from particle acceleration around the periphery of the electromagnetic jet.

\section{Calibration of BH magnetic energy output into the IGM}

\subsection{Quantification of BH energy release into the IGM}

The following graph, reproduced from Kronberg et al. (2001) shows measured total energy contents of lobe images for a large sample of giant radio galaxies (GRG), and compares these with the (gravitational) formation energy of the host galaxy's central black hole, taking $10^{8} \mathrm{M}_{\odot}$ as a fiducial $\mathrm{BH}$ mass. The upper envelope of GRG energy contents is, remarkably, within about an order of magnitude of the putative gravitational energy reservoir. This verifies the typical high efficiency of energy conversion from gravitational to magnetic $\eta_{B}$ (and particle, $\eta_{P}$ ) energy (Kronberg et al. 2001). Radio lobes approximately within a cluster core radius (open squares in Fig. 4) have markedly lower internal energies, and the energy deficit, compared with the GRG's, is approximately the $P \mathrm{~d} V$ work done against the intracluster gas, whose pressure can be independently estimated from the intracluster X-ray emission!

\subsection{Basic calculations of expectation}

The average density of supermassive black holes is now fairly well known, and shown to correlate with the bulge mass of the host galaxy. Given this average intergalactic $\mathrm{BH}$ density, $\rho_{\mathrm{BH}}$, we expect that, sometime within a Hubble time, every central $\mathrm{BH}$ releases an enormous amount of magnetic energy $\sim 10^{61-62}$ ergs into the IGM via a jetlobe system. Thus, globally over a few $\times 10^{9}$ yr this magnetic energy diffuses to an intergalactic volume.

$$
\begin{aligned}
& <\rho_{B H}>\approx 2 \times 10^{5} M_{\odot} / M p c^{3} \\
& M_{B H} c^{2}=1.8 \times 10^{62} \frac{M_{B H}}{10^{8} M_{\odot}} \text { ergs }
\end{aligned}
$$

where $M_{B H} c^{2}$ is the gravitational energy per BH associated with infall down to the BH's Schwarzschild radius. Let us now assume that a fraction, $f_{\text {FILAMENTS }}^{V O L}$ of the entire IGM volume is available to be filled by magnetic flux from aggregate magnetic energy outflow. The available volume constitutes the filaments and walls of galaxy overdensity in large 


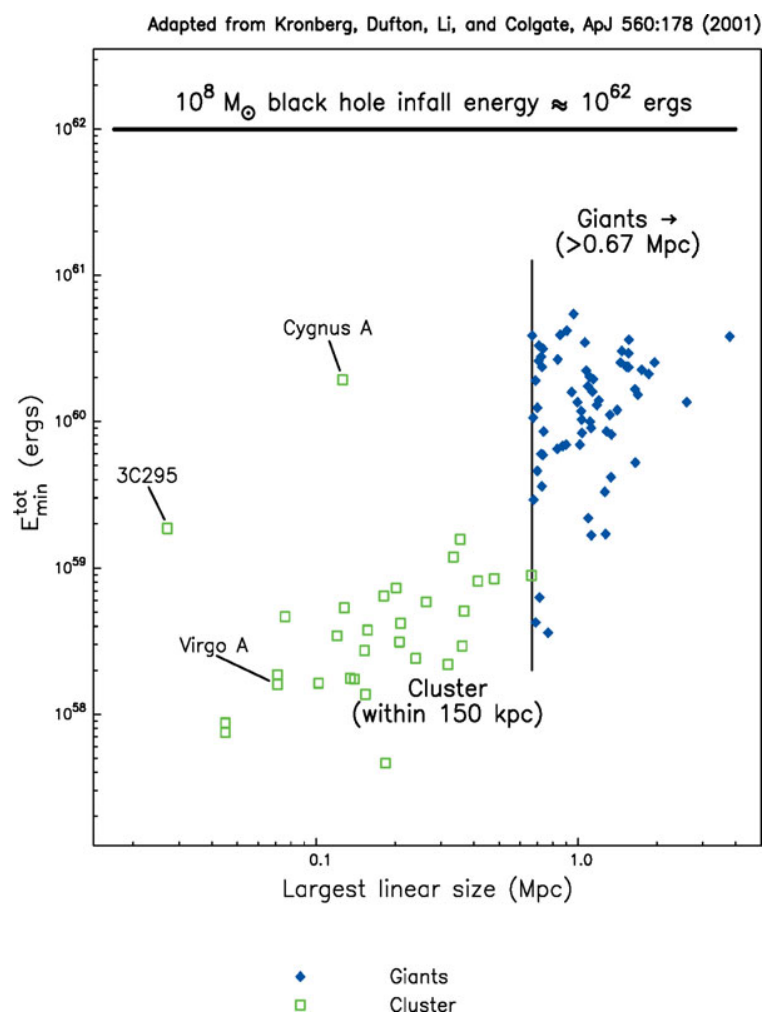

Figure 4. The total radio lobe energy content for (a) a 70-source sample of giant radio galaxies, and (b) for radio lobes within $150 \mathrm{kpc}$ of the centre of a galaxy cluster. For sample (a) a lower size cutoff of $700 \mathrm{kpc}$ was applied. Reproduced from Kronberg et al. (2001).

scale structure (LSS).

$$
\varepsilon_{\mathrm{B}}=1.36 \times 10^{-15}\left(\frac{\eta_{\mathrm{B}}}{0.1}\right) \times\left(\frac{f_{\mathrm{RG}}}{0.1}\right) \times\left(\frac{f_{\mathrm{FILAMENTS}}^{\text {VOL }}}{0.1}\right)^{-1} \times\left(\frac{M_{\mathrm{BH}}}{10^{8} M_{\odot}}\right) \mathrm{erg} \mathrm{cm}^{-3}
$$

The fraction of $M_{B H} c^{2}$ released as magnetic energy is $\eta_{B}$, and fraction of all elliptical galaxies that become radio sources at some point in their lifetime is defined as $f_{\mathrm{RG}} \cdot \varepsilon_{\mathrm{B}}$ is the average injected intergalactic magnetic energy density. The corresponding magnetic field strength is

$$
B_{I G}^{B H}=\sqrt{8 \pi \varepsilon_{\mathrm{B}}}=1.8 \times 10^{-7} \mathrm{G}
$$

for the normalization chosen in (4.2). This estimate is high enough to have some chance of being observable, by exploiting the capabilities of current instruments. I discuss this in the next section.

\section{New attempts to verify BH magnetic energy "sinks" in the IGM}

This section describes two attempts to detect intergalactic magnetic fields that might have been infused by central BH's. In the first, $B_{I G}^{B H}$ would be revealed in faint diffuse synchrotron radiation. In the second, we searched for a Faraday rotation signal due to a hypothesized $B_{I G}^{B H}$ in a nearby, large supergalactic filament - the Perseus-Pisces supercluster. 


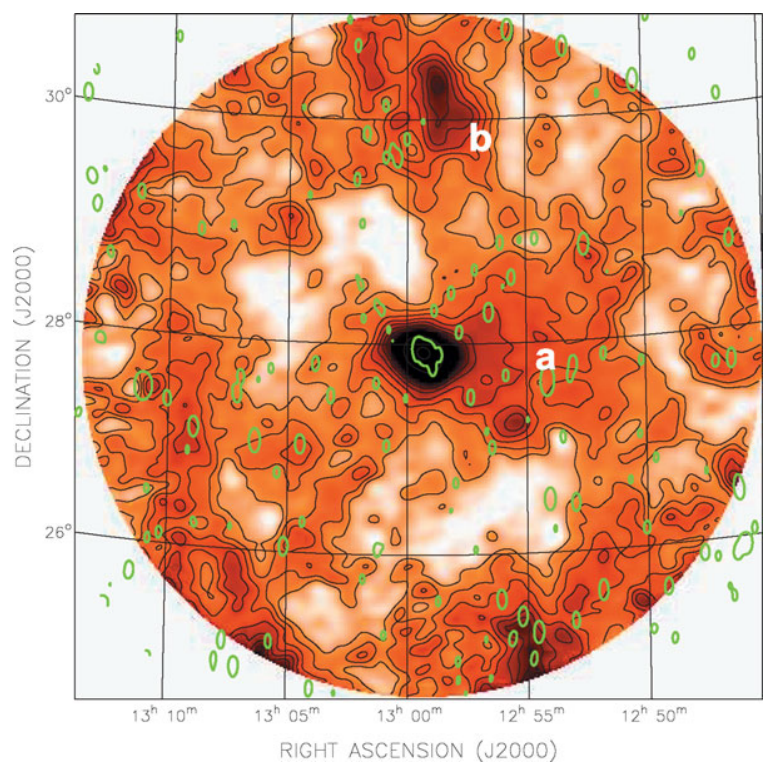

Figure 5. Diffuse radiation at $0.4 \mathrm{GHz}$ from the first combination of the Arecibo Telescope with the DRAO Interferometer. The resolution of this image is 10' - that of the Arecibo $305 \mathrm{~m}$ telescope. The $2.7 \mathrm{~K} \mathrm{CMB}$, and discrete sources have been removed, as has a linear-plane component of the Milky Way foreground radiation. Ellipses (proportional to source flux density)show the locations of the strongest discrete sources that were removed. $T_{b}$ contours are shown at 1.4, 1.9, $2.4,2.9,3.4,3.9,4.4,10$, and $40 \mathrm{~K}$. The rms noise is $250 \mathrm{mK}$, and is limited by confusion. Thermal noise is $\sim 4 \mathrm{mK}$. Adapted from the colour original in Kronberg, Kothes, Salter, \& Perillat (2007).

\subsection{Diffuse synchrotron radiation detected with an unprecedented combination of $T_{b}$ sensitivity and resolution}

The detection of a zone of extragalactic magnetic field by synchrotron radiation requires a very high instrumental surface brightness sensitivity, and a resolution that is sufficiently high to ensure that the emission is diffuse, and not a blend of faint discrete radio sources. Finally, since faint diffuse radiation patches can also be expected from Milky Way foregrounds, some way is needed to plausibly ascertain if the diffuse emission in question truly intergalactic.

A combined Arecibo-DRAO Interferometer image of a $9^{\circ}$ field within the Coma supercluster at $\simeq 100 \mathrm{Mpc}$ distance is shown in Figure 5. Here, the output of deep scans with the 10' beam of the Arecibo telescope were Fourier transformed into $u-v$ space, and combined with double, full synthesis observations of the same wide field with the DRAO Interferometer (Kronberg et al. 2007). The full resolution of the resulting combined image $\simeq 3.5^{\prime}$, that of the DRAO interferometer.

The area "a" contains widespread, diffuse glow that appears "connected" to the strong synchrotron halo of the Coma cluster itself. Within its projected size of $2 \mathrm{Mpc}$, this feature contains about 7 low luminosity radio galaxies and all have redshifts comparable with the Coma cluster. This large region has comparable dimensions to a giant radio galaxy, but it is not associated with any single galaxy. Its synchrotron luminosity can be interpreted as due to the collective energization of several AGN-powered radio sources. The total energy content of region "a" is $\sim 10^{60} \mathrm{ergs}$, and $B_{I G}$ is $10^{-7} \mathrm{G}$ or greater, both of which are consistent with this interpretation. The unusually high concentration of radio galaxies in the Coma region of the "Great Wall" supercluster enables a kind of test 


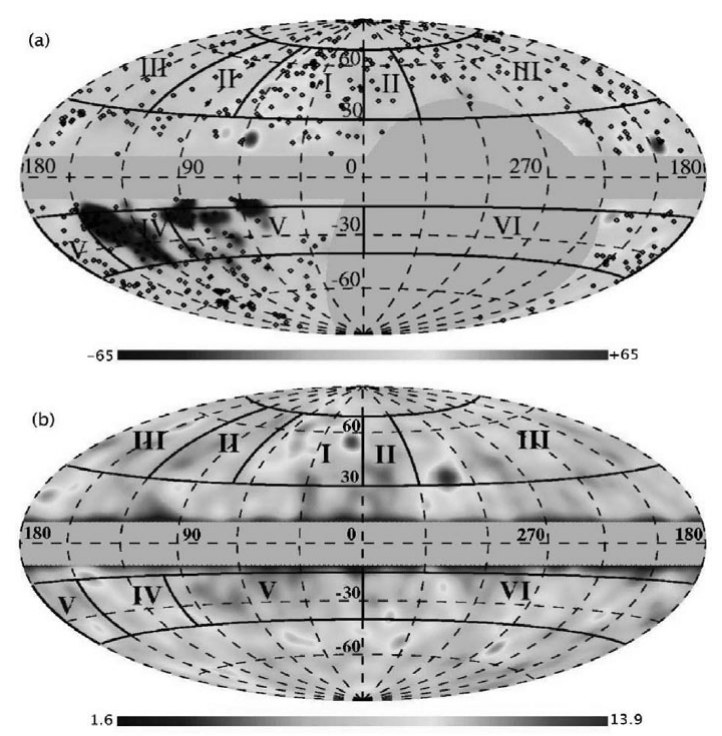

Figure 6. Smoothed extragalactic source RM's (upper), and smoothed 2MASS galaxy column densities (lower) compared at the same resolution of $7^{\circ}$. The p-p supercluster "chain" is approx. between $l 120^{\circ}$ and $150^{\circ}$, and from $b-18^{\circ}$ to $-50^{\circ}$. Further details can be found in Xu et al. (2006).

laboratory for equation (4.2). It is likely the first direct confirmation of the magnetization of the IGM due to a collectivity of radio galaxies. For further details, and discussion of other new radio features in Figure 5 see Kronberg et al. (2007).

\subsection{Search for a detectable $B_{I G}^{B H}$ via Faraday rotation in a nearby filament of galaxy overdensity}

As with the synchrotron glow test for intergalactic fields at the $10^{-7} \mathrm{G}$ level, A Faraday RM test also presses the limits of current technology. Using a suitable subset of extragalactic source RM's with a high measurement accuracy, Xu et al. (2006) compared the RM's with the $(l, b)$ - dependent galaxy densities in the 2MASS galaxy survey, and separately for the CfA2 survey which had spectroscopic redshifts. The more accurate $z$ 's of the CfA galaxies permitted construction of 3-D intergalactic zones of galaxy overdensity, and the galaxy density - weighted pathlengths through these supercluster volumes. For the less accurate $z$ 's of the 2MASS survey, corresponding galaxy column densities were calculated in an independent exercise.

$\mathrm{Xu}$ et al. 2006 investigated 3 superclusters at locations suitably far from the higher RM zones of the Galactic plane. These were the Virgo, Hercules and Perseus-Pisces superclusters, and the test was to ascertain if any excess Faraday rotation could be seen through the supercluster volumes. The Virgo supercluster is too dilute in the sky, and the Hercules supercluster was subject to confusion with higher latitude Galactic RM features. The galaxy distribution in the Perseus-Pisces supercluster is the most clearly defined, and probably, though not with absolute certainty, less confused by a Galactic foreground RM. Assuming the latter not to be the case, $\mathrm{Xu}$ et al. found evidence for $B_{I G}$ of few $\times 10^{-7} \mathrm{G}$ for assumed magnetic field reversal scales of $200-800 \mathrm{kpc}$. The fields must be scaled, of course, to $n_{I G}$ the intergalactic electron density. Values of $n_{I G}$ on these scales are still very uncertain, but were assumed to be a few $\times 10^{-5} \mathrm{~cm}^{-3}$ on the basis of X-ray absorption and EUV -based estimates. More, and comparably accurate RM's, 
and more detailed galaxy counts and spectra are needed to carry this type of probe a stage further in the future. However these $B_{I G}$ values are independently consistent with equation (4.2).

\section{Conclusions}

In these few pages I have selected some recent computational and observational results that appear to outline a self-consistent physical picture of the physics of magnetized extragalactic jets and radio/X-ray lobes. I have argued that current dominated jets and lobes, as simulated in several recent papers are coupled to each other, and that they are poynting flux dominated. I also reviewed their global energetics, with the conclusion that central BH's alone contribute much of their enormous formation energy to intergalactic space. They contribute significantly to a magnetized intergalactic medium.

\section{Acknowledgements}

I thank Stirling Colgate, Hui Li, and Masanora Nakamura for beneficial discussions, and Raul Cunha, Quentin Dufton, and Phyllis Orbaugh for their assistance. I also thank the Natural Sciences and Engineering Research Council of Canada (NSERC) and the U.S. Department of Energy for support.

\section{References}

Benford, G. \& Protheroe, R. J. 2008, MNRAS 383, 663

Blandford, R. D. \& Znajek, R. L. 1977, MNRAS 197, 433

Bridle, A. H., Hough, D. H., Lonsdale, C. J., Burns, J. O., \& Laing, R. A. 1994, AJ 108, 766

Li, H., Colgate, S. A., Wendroff, B., \& Liska, X. Z. 2001, ApJ 581, 874

Diehl, S., Li, H., Fryer, C. L., \& Rafferty, D. 2008, ApJ, in press

Kataoka, J., Edwards, P., Georganopoulos, M., Takahara, F., \& Wagner, S. 2003, A 8 A 399, 91

Kovalev, V. V., Lister, M. L., Homan, D. C., \& Kellermann, K. I. 2007, ApJ 668, L27

Kronberg, P. P. 1976a, ApJ 203, L47

Kronberg, P. P. 1976b, I.A.U. Symp. 74

Kronberg, P. P., Burbidge, E. M., Smith, H. E., \& Strom, R. G. 1977, ApJ 218, 8

Kronberg, P. P. 1986, Can.J. Phys. 64, 449

Kronberg, P. P., Dufton, Q. W., Li, H., \& Colgate, S. A 2001, ApJ 560, 178

Kronberg, P. P., Kothes, R., Salter, C. J., \& Perillat, P. 2007, ApJ 659, 267

Lapenta G. \& Kronberg, P. P. 2005, ApJ 625, 37

Leahy, P. \& Perley, R. A. 1991, AJ 102, 537

Lesch, H., Appl, S., \& Camenzind, M. 1989, A\&A 225, 341

Li, H., Lapenta, G., Finn, J. M., Li, S., \& Colgate, S. A. 2006, ApJ 643, 92

Lovelace, R. V. E. 1976, Nature 262, 649

Nakamura, M., Tregillis, I. L., Li, H., \& Li, S. 2008, ApJ 686, 843

Owen, F. N., Biretta, J., \& Eilek, J. 1989, ApJ 698, 707

Robinson, K., Dursi, L. J., Ricker, P. M., Rosner, R., Calder, A. C., Zingale, J. W., Truran, J. W., Linde, T., Cacares, A., Fryxell, B., Olson, K., Riley, K., Siegel, A., \& Vladimirova, N. 2004, ApJ 601, 623

Wise, M. W., McNamara, B. R., Nulsen, Houck, P. E. J., \& David, L. P. 2007, ApJ 659, 1153

Xu, Y., Kronberg, P. P., Habib, S., \& Dufton, Q. W. 2007, ApJ 637, 19 


\section{Discussion}

DurRer: Are the magnetic fields in PP super clusters coherent? On which scales?

KRONBERG: If confirmed, they must have a large coherent component, up to $\approx 800 \mathrm{kpc}$.

DE GeOuveia dal Pino: There is a poster upstairs where we have explored the role of SN-turbulent feeding on the ICM central region of the Perseus cluster and we have found that this can be a competing and even a dominant effect when compared to the AGN feedback (Falceta-Gonçalves, de Gouveia dal Pino, Gallagher, Lazarian (Poster 1.10, this conf.). Could you comment on the relative importance of these two mechanism for the central ICM structure and evolution?

KRONBERG: That is entirely possible, I agree, especially in the ICM. I avoided star/SNdriven outflow in my talk only to restrict my focus to massive-BH driven energy flows within the limited time.

SCHLICKEISER: 1) Unless there is a return current, your quoted outflow current of $\approx 10^{18}$ violates the Alfven current limit by 14 orders of magnitude. 2) The presence of a pair outflow would naturally explain the small Faraday RM in 3C303. Why did you exclude this interpretation? 3) If the Alfven speed is of order $v_{\mathrm{A}} \simeq c$ you can not form superAlfvenic shock waves to explain your lobes.

Kronberg: 1) Yes, there is a return current, but for 3C303 it is likely more diffuse, and outside $r \approx 250 \mathrm{pc}$ from the jet axis. Certain variations of the CDJ models could, however, have the return current closer to the axis than in the 3C303 system. 2) Possibly, but not the $\nabla \mathrm{RM}$ measurement. 3) The electron acceleration in the highly magnetized lobes might have other causes. However, I only discussed $v_{\mathrm{A}}$ in the jet "cocoon" zone.

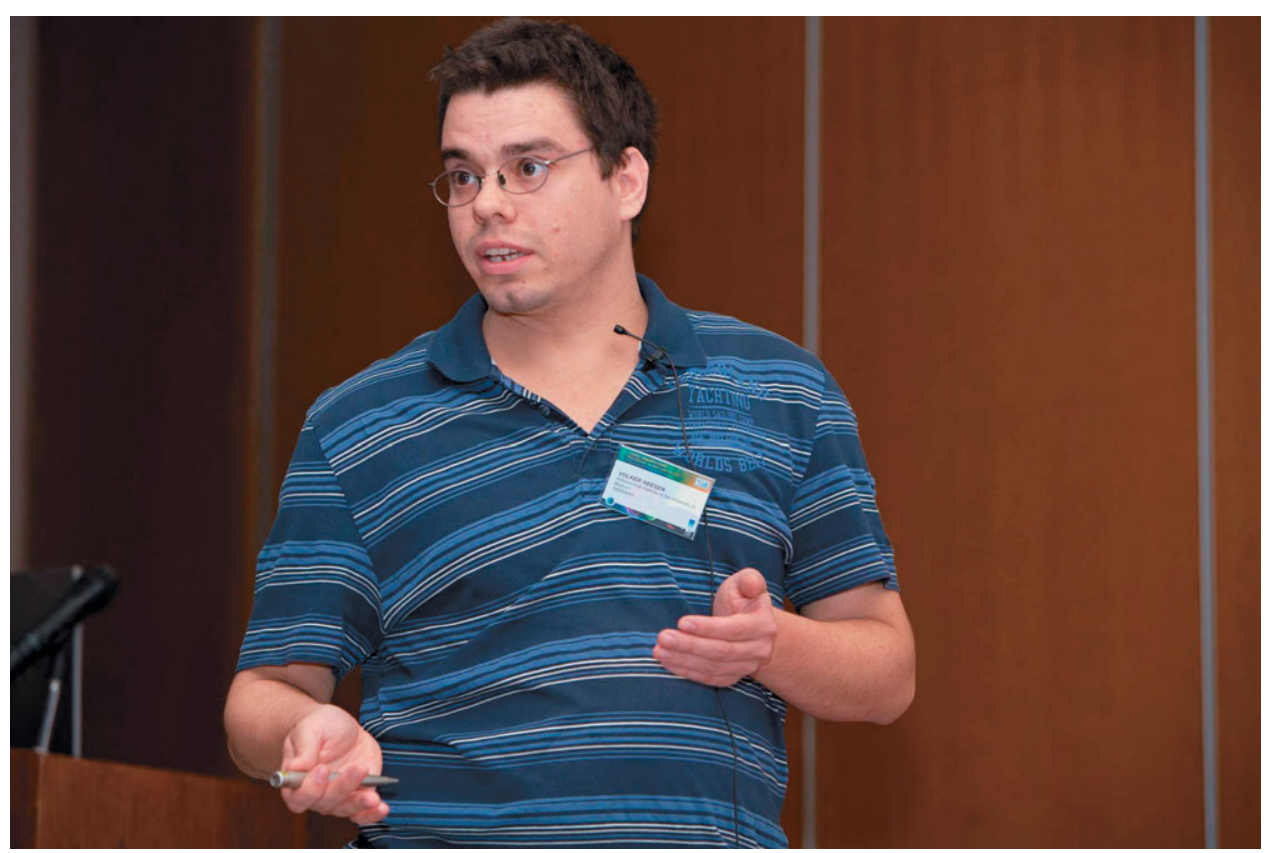

Volker Heesen 